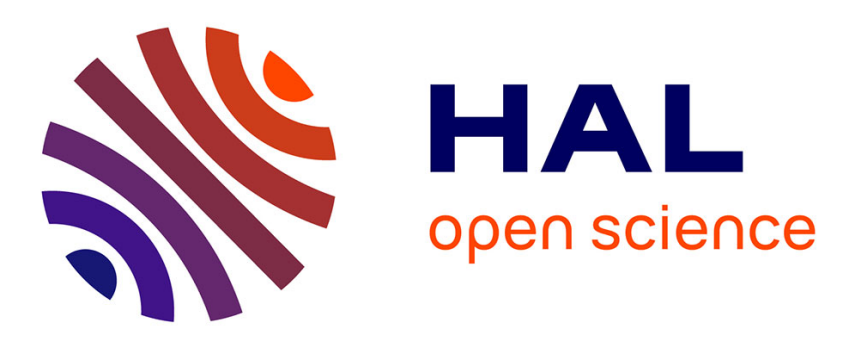

\title{
Solar wind decrease at high heliographic latitudes detected from Prognoz interplanetary lyman alpha mapping
}

\author{
Rosine Lallement, Jean-Loup Bertaux, V. G. Kurt
}

\section{To cite this version:}

Rosine Lallement, Jean-Loup Bertaux, V. G. Kurt. Solar wind decrease at high heliographic latitudes detected from Prognoz interplanetary lyman alpha mapping. Journal of Geophysical Research Space Physics, 1985, 90 (A2), pp.1413-1423. 10.1029/JA090iA02p01413 . hal-02889795

\section{HAL Id: hal-02889795 https://hal.science/hal-02889795}

Submitted on 16 Mar 2021

HAL is a multi-disciplinary open access archive for the deposit and dissemination of scientific research documents, whether they are published or not. The documents may come from teaching and research institutions in France or abroad, or from public or private research centers.
L'archive ouverte pluridisciplinaire HAL, est destinée au dépôt et à la diffusion de documents scientifiques de niveau recherche, publiés ou non, émanant des établissements d'enseignement et de recherche français ou étrangers, des laboratoires publics ou privés. 


\title{
Solar Wind Decrease at High Heliographic Latitudes Detected From Prognoz Interplanetary Lyman Alpha Mapping
}

\author{
R. Lallement ANd J. L. BertauX \\ Service d'Aéronomie du CNRS, Verrières-le-Buisson, France \\ V. G. KURT \\ Space Research Institute, Academy of Science of USSR, Moscow
}

\begin{abstract}
New evidence for a latitudinal decrease of the solar wind mass flux is presented from observations of the interplanetary Lyman alpha emission collected in 1976 and 1977 with satellites Prognoz 5 and 6. The flow of interstellar hydrogen atoms in the solar system is ionized by EUV solar radiation and charge exchange with solar wind protons which accounts for about $80 \%$ of the total ionization rate. The resulting gradual decrease of the neutral $\mathbf{H}$ density from the upwind region down to the downwind region observed from Ly $\alpha$ intensity measurements allowed the determination of the absolute value of the total ionization rate $\beta$ for one $\mathrm{H}$ atom at $1 \mathrm{AU}$ against ionization. Collected in 1976 and 1977 at five places in the solar system, the.measurements are first compared to a model which assumes isotropy of the EUV and solar wind. Strong departures are obvious toward high-latitude regions, especially when the observer is in the downwirid region where the solar wind ionization has had more time to act (cumulative effect). A model was constructed which included a decrease of the ionization rate with heliographic latitude. The adjustment of data allowed for the measurement of the absolute value of the total ionization rate and implies a $50 \%$ latitude decrease of the ionization rate due to charge exchange with the solar wind, from $\beta_{s w}=(3.9 \pm 0.5) \times 10^{-8} \mathrm{~s}^{-1}$ at the equator to $\beta_{s w}=(2.0 \pm 0.5) \times 10^{-8} \mathrm{~s}^{-1} \mathrm{at}$ the pole. The corresponding absolute value of the solar wind proton flux is $(2.4-3.6) \times 10^{8} \mathrm{~cm}^{-2} \mathrm{~s}^{-1}$ at the equator and twice less at the pole if a constant velocity is assumed for the solar wind. Even if the solar wind velocity increases from 400 to $800 \mathrm{~km} \mathrm{~s}^{-1}$, which would decrease the charge exchange cross section by $25 \%$, there is still a decrease by about $30 \%$ of the solar wind mass flux from equator to pole. The Lyman alpha data from Mariner 10 (Kumar and Broadfoot, 1978) had already shown a similar trend in 1974, showing a persistence of the solar wind anisotropy for 3-4 years during a solar minimum. The large-scale properties of the solar wind mass flux can therefore be monitored at all latitudes by remote sensing of $\mathrm{Ly} \alpha$ interplanetary emission, since the solar wind is carving the flow of interstellar $\mathbf{H}$ and its anisotropies are "printed" on the interplanetary $\mathrm{H}$ distribution. With uncalibrated Ly $\alpha$ measurements, an absolute value of the solar wind flux can be determined at all latitudes averaged over a typical 1-year period.
\end{abstract}

\section{INTRODUCTION}

It has been shown through modeling by Joselyn and Holzer [1975] that if there is a nonisotropic source of the solar wind, the spatial distribution of atomic hydrogen in the interplanetary space would be greatly affected, which could be observed by remote sensing with a mapping of the interplanetary Ly $\alpha$ emission produced through resonance scattering of Ly $\alpha$ solar photons.

More recently, Kumar and Broadfoot [1979] have observed and analyzed such an effect on the Mariner 10 results. In the present paper we show clear evidence of the nonisotropic character of the solar wind ionization effect from observations of the Ly $\alpha$ interplanetary emission collected with Prognoz 5 and 6 Soviet spacecraft in 1976 and 1977.

Few other recent observations of the solar wind properties have shown latitudinal dependences of the plasma densities and velocities. From analysis of both in situ measurements and interplanetary scintillations (IPS) observations, latitude gradients in the velocities have been derived [Rhodes and Smith, 1976; Rickett and Coles, 1979; Zhao and Hundhausen, 1983], even at high latitude [Coles and Maagoe, 1972]. In general, high velocities are associated with high latitudes, but they also coincide with equatorial coronal holes or with polar coronal holes and their lower latitude extensions.

Copyright 1985 by the American Geophysical Union.

Paper number 4A8193.

0148-0227/85/004A-8193\$05.00
The spatial evolution of the coronal holes which are associated with low plasma densities and open magnetic field lines has been deduced from ground-based white light coronal measurements [Hundhausen et al., 1981]. The most extended features are the polar coronal holes appearing at low solar activity. In the case of near-ecliptic observations, some results about the solar protons fluxes have been obtained in situ by Schwenn [1982] showing a decrease of the flux in the highspeed streams which are associated with open magnetic field lines.

Up to now there have been many local and unrelated results, but a general description of the latitude dependence is still lacking, and there is a need for out of ecliptic measurements, especially of the density fluxes. The International Solar Polar Mission, scheduled to pass over the solar poles in 1990 , will certainly gather numerous in situ measurements at high latitudes.

However, the remote sensing monitoring of the interplanetary Ly $\alpha$ emission provides a way to determine properties of the solar wind with a large spatial scale, averaged over time scales of the order of 1 year, by using its ionizing effect on the interplanetary-interstellar hydrogen gas.

\section{InTERPLANETARy Lyman Alpha Method}

Because of its relative motion in respect to the local interstellar medium, characterized by the velocity vector $\mathbf{V}_{w}=$ $-V_{0}$, the solar system is filled in by a permanent flow of neutral hydrogen and helium atoms of interstellar origin. 


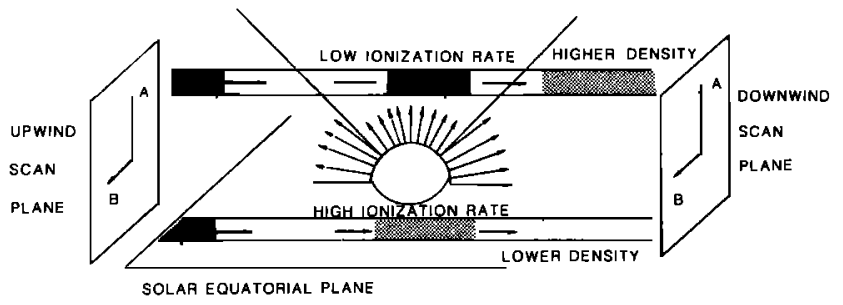

Fig. 1. Schematic illustration of the interaction between the hydrogen flow and an anisotropic solar wind. For the sake of simplicity the hydrogen bulk velocity is assumed to be in the solar equatorial plane. The exact configuration is shown in Figure 10 and has been taken into account in the modelling. Atoms which travel near the solar equatorial plane do suffer higher loss processes than the atoms which are passing over the polar regions. Thus the density decrease is larger in the first case, and there are regions of enhanced density above and below the ecliptic plane. The discrepancies between the intensities in the directions $A$ and $B$ will be much more pronounced in a downwind scan plane than for directions $A^{\prime}$ and $B^{\prime}$ in an upwind plane of observation, since downwind the distribution has been significantly altered by the cumulated effects along the wind flow.

Atoms which travel in the vicinity of the sun are submitted to the ionizing EUV radiation and corpuscular solar fluxes. For hydrogen, the most important ionizing effect is charge exchange with solar protons (about $80 \%$ of the whole loss processing), followed by EUV photoionization ( $\lambda \leq 91.2 \mathrm{~nm}$ ) $(\simeq 20 \%)$, while charge exchange with alpha particles and ionization by collisions with the solar electrons have been estimated of very minor importance [Holzer, 1977]. While photoionization leads to the disparity of the neutrals, charge exchange between a "slow" interstellar $\mathrm{H}$ atom $\left(V_{0}\right.$ is about 20 $\left.\mathrm{km} \mathrm{s}^{-1}\right)$ and a fast solar proton $\left(\simeq 400 \mathrm{~km} \mathrm{~s}^{-1}\right)$ produces a slow new proton and a fast neutral. However, these new fast atoms do not contribute to the interplanetary Ly $\alpha$ emission for the following two reasons. Because of their large radial velocities, their density is much lower than the initial neutrals (according to the velocity ratio, about 20 times less); but most important, owing to their large Doppler effect they are only very weakly illuminated by the very far blue wing of the solar Ly $\propto$ line, which is less than $2 \%$ of the intensity at line center for a velocity of $400 \mathrm{~km} \mathrm{~s}^{-1}$. Thus the resonantly scattered interplanetary Ly $\alpha$ emission represents practically exclusively the slow neutral population of atoms which have not yet suffered ionization. The flow of these neutrals is progressively affected by the above mentioned loss processes, more and more along the "downwind" direction, (direction of the vector $V_{0}$ ). In the theoretical case of a complete spherical symmetry of the solar fluxes, the hydrogen density, the velocity, and the emissivity distributions also present a symmetry of revolution around and axis containing the sun and the velocity vector $\mathbf{V}_{\mathbf{0}}$. Thus departures from this axial symmetry can be attributed to anisotropies of solar properties. Some such departures have already been observed with the Mariner 10 spectrophotometer on Ly $\alpha$ results [Kumar and Broadfoot, 1978, 1979; Witt et al., $1979,1981]$ and have been interpreted by these authors as the result of a decreasing solar wind flux with higher ecliptic latitude. The present analysis has led to similar conclusions, with somewhat different numerical determinations of the degree of anisotropy. Improvements of this analysis in respect to Mariner 10 results include a better statistical accuracy (larger data set) and a greater variety in the geometries of observation, which is quite important as it will be seen below. Here the model takes into account the full velocity distribution, and some parameters of the model were determined from the hy- drogen absorption cell data, whereas they were guessed in Mariner 10 análysis.

In Figure 1 the effect of the anisotropic interaction between solar wind fluxes and the hydrogen flow is represented in a schematic way. The thermal velocity dispersion inside the flow was neglected, and $V_{0}$ was assumed to be in the solar equatorial plane for simplicity. Let us suppose that atoms following trajectories passing over the polar regions encounter a solar plasma flux with a less ionizing power compared with the low-latitude plasma flux acting on the atoms which travel near the equatorial plane. Thus owing to the different ionization rates, the density decrease along the polar trajectory and departures from the axisymmetry appear gradually from the upstream region, where the flow is uniform down to the downstream space where the distribution presents two regions of enhanced density, perpendicularly above and below the ecliptic plane. From this simple scheme it appears that the observer's position is of great importance. The maximum contrast for intensities resulting only from solar ionization anisotropies is for an observer on the downwind side of the sun, when he looks, for instance, in a plane normal to $V_{0}$. One of the Mariner 10 geometry of observations was not far from this ideal case. This is also the reason why we have selected for analysis two periods of observations obtained with Prognoz $6 \mathrm{Ly} \alpha$ photometer, when the earth was very near the downwind axis. At variance with some other potential sources of emission anisotropy acting on the $\mathrm{Ly} \alpha$ excitation rate of atoms (like a nonisotropic Ly $\alpha$ solar flux), the ionization anisotropy is cumulated along the wind flow, as well as the resulting emission anisotropy. One period of observation covered 15-40 hours of data and will be referred to as an observation in the following text.

It would have been useful, as we will discuss in section 3 , to have also a corresponding scan plane on the upwind side, to make sure of the unique role of the solar wind by monitoring the contrast evolution along the flow. Unfortunately, we have no results collected when the earth was in the upwind region, but despite of this we could use other earth positions and scan planes giving upwind observations (see Figure 2) in order to check that the gradual evolution described above was effectively contained in the results.

Although the solar wind anisotropies are revealed by photometric measurements and a corresponding photometric model, the advantage of the Prognoz measurements is the conjunction of two types of diagnostic of the interplanetary hydrogen, a spectral scanning with a hydrogen absorption cell yielding the velocity distributions of $\mathrm{H}$ atoms [Bertaux and Lallement, 1984] and a photometric mapping. The reason is that there are large uncertainties on the determination of the model parameters if only photometric results are used. As a matter of fact, the general decrease of the density along the flow (independently of the anisotropies) is determined at the same time by 4 parameters: the mean ionization rate $\beta$, the velocity modulus $V_{0}$, the temperature $T$ of the gas at "infinity," and the dynamical effect of the Ly $\alpha$ radiation pressure represented by the parameter $\dot{\mu}$, the ratio of radiation pressure to gravitation. A same Ly $\alpha$ emission pattern may result from an infinite number of sets of parameters $\beta, V_{0}, T$, and $\mu$ as calculated for instance by $R$. Lallement and J. L. Bertaux (unpublished manuscript, 1984). Up to now, at least two of these parameters were fixed a priori when analyzing Ly $\alpha$ photometric measurements. However, it is of great importance when spatial variations of the ionization rate are detected to use the good set of parameters in order to achieve an accurate measurement of 


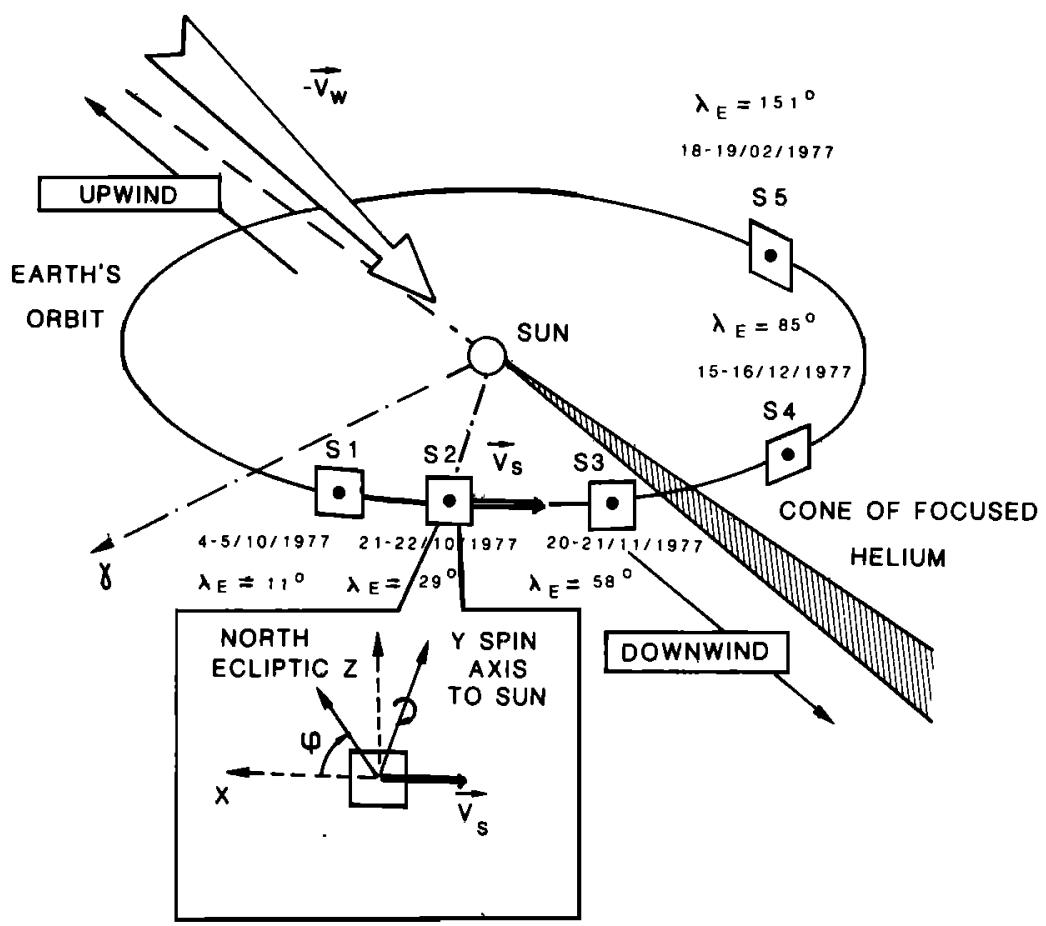

Fig. 2. Five scan planes from the Prognoz 5 and 6 measurements are shown along the earth's orbit. Each plane is perpendicular to the sun-earth line. The helium cone has been represented and indicates the position of the downwind direction. In a scan plane, the spin angle $\phi$ for a line of sight is counted from axis $X$ opposite from the earth's velocity vector $\mathbf{V}_{s}$. The date and ecliptic longitude is indicated for each observation.

the anisotropy. The hydrogen absorption cell associated with the Ly $\alpha$ photometer in the Prognoz experiment acting like a high resolving power spectrometer allowed for the first time an accurate determination of the dynamical parameters $V_{0}, \mu$, and $T$ [Lallement, 1983; J. L. Bertaux et al., unpublished manuscript, 1984] without using the measured intensities. Thus the photometric pattern can entirely be devoted to the study of ionizing effects, since there is no more ambiguity over the parameters of the model.

In the present analysis a series of positions for observations was used allowing both the detection of the largest contrast (downwind position) and a comparison of the contrasts from the upwind to the downwind observed regions.

Comparison of Prognoz intensities with the isotropic model which depends only upon one parameter, the ionization rate $\beta$, shows that there are, whatever the value of $\beta$, systematic (Figures $3 a-3 e$ and Figure 4) enhancements of the intensity around the north and south ecliptic directions, which are near the directions normal to $\mathbf{V}_{0}$. Thus attributing these departures to a nonspherical ionization effect, we constructed a nonisotropic model described in section 4. However, before discussing the results of this nonisotropic model it is necessary to examine critically in the next section the other possibilities which could also result in some departures to the spherical model and compare their efficiency with the nonisotropic solar wind explanation.

\section{Charge Exchange With Solar Protons} as a Principal Source of Departures With AXYSYMMETRIC HYDROGEN EMISSIVITY DisTRIBUTION

What is observed initially when an isotropic (or spherical) model of solar interaction is taken is a disagreement between measured and predicted intensities. As possible inconsistencies with the isotropic model hypothesis we could propose (1) an extrainterplanetary contribution, (2) an initial inhomogeneous flow of gas, (3) multiple scattering influences, (4) anisotropy of the illuminating solar Ly $\alpha$, (5) anisotropy of the solar EUV fluxes, and (6) anisotropy of the solar wind. These inconsistencies are discussed below.

1. The resonance scattering of the solar Ly $\alpha$ line by the interplanetary hydrogen has been shown to constitute almost the totality of the extraterrestrial Ly $\alpha$ signal. An upper limit for the galactic or extragalactic contribution has been given from a theoretical point of view [Thomas and Blamont, 1976] and has been experimentally deduced [Bertaux et al., 1976; $\mathbf{R}$. Lallement and J. L. Bertaux, unpublished manuscript, 1984]. This last limit of 15 rayleigh is largely smaller than the observed discrepancies of intensities between the best possible spherical model and the data. In addition, directions of enhanced emissivity coincide with high ecliptic (approximate heliographic latitudes) and not with the galactic disk at all.

2. Perturbations of the neutral part of the interstellar medium at the heliospheric boundary could introduce inhomogeneities in the incoming gas. However, since the distances from the sun to the heliopause are evaluated to be of the order of at least $100 \mathrm{AU}$, one would expect a spatial scale of variations greater than the dimensions of the volume which contributes, in fact, to the detected emission. As a matter of fact, owing to the $r^{-2}$ radial decrease of the solar Ly $\alpha$ incident flux, dimensions of the emitting volume are of the order of 5 (upwind side) to 10 (downwind side) AU.

In addition, if inhomogeneities of the distribution exist before passing by the sun, their amplitudes should be identical on the upwind and downwind side, and the model which is presented in the next section which takes into account a gradual apparition of anisotropies should not have allowed the same good agreement for both upwind and downwind directions. 


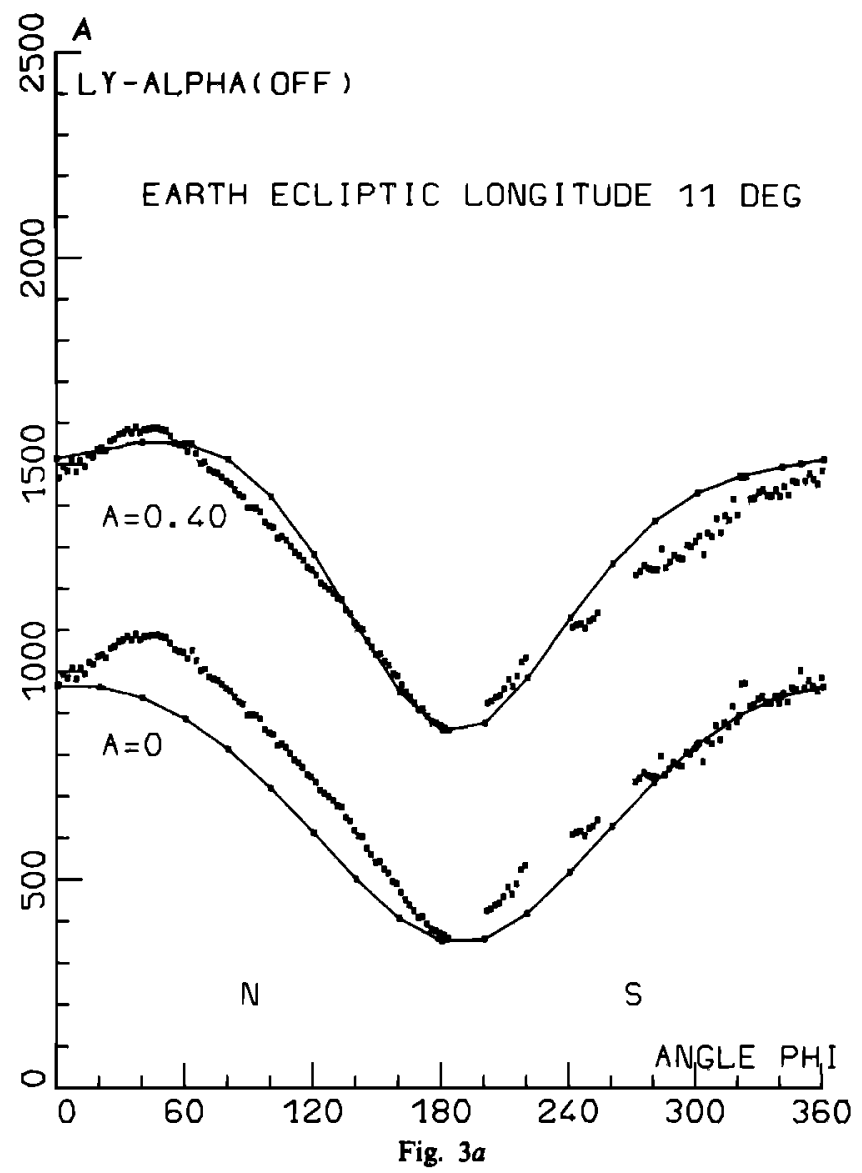

Fig. 3. Measurements of the interplanetary Lyman $\alpha$ emission (points) are represented as a function of spin angle $\phi$ for the five observations at different ecliptic longitudes and are compared to two different models of the solar wind effect. For the sake of clarity, data points are plotted twice on each figure. The ordinate scale in counts per second is valid for the lower set of data points compared to a model assuming an isotropic solar wind $(A=0)$. The same data points are displaced upward by 500 counts $/ \mathrm{s}$ and are compared to a model including anisotropy of the solar wind characterized by the parameter $A=0.40$. The north and south ecliptic directions are indicated by the letters $\mathbf{N}$ and $\mathbf{S}$. The introduction of an anisotropic ionization rate characterized by $40 \%$ of relative diminution from the equatorial to polar regions allows the best fit of the directions of maximum intensity for each observation and the overall shape of the curves as a function of $\phi$. In particular, for observations near the downwind axis $\left(\lambda_{e}=58\right.$ and $85^{\circ}$, Figures $3 c$ and $\left.3 d\right)$, the isotropic model shows only one maximum and one minimum, whereas both data and the anisotropic model show two maxima. In respect to the isotropic model the data points show more Lyman $\alpha$ emission toward the ecliptic pole, showing that the solar wind was less effective to destroy $\mathrm{H}$ atoms at high ecliptic latitudes. For observations $S_{1}$ to $S_{4}$ (Figures 3a-3d) the calibration factor for Prognoz 6 measurements was estimated to be 3.7 counts $\mathrm{s}^{-1}$ rayleigh $^{-1}$, as determined from a UV star observation. According to this value the adjustment of the model to the data is consistent for the four observations and corresponds to a density of infinity $n_{\infty} \simeq 0.04$ atom $\mathrm{cm}^{-3}$. The calibration factor for the last observations $S_{5}$ is about 2.1 counts rayleigh ${ }^{-1}$ (Prognoz 5).

3. The present model has been constructed using the assumption of an optically thin medium. If the importance of the multiple scattering effects is not negligible, as it was calculated by Keller et al. [1981], it does not affect the axial symmetry of the emissivity and thus it cannot be invoked as a possible explanation of the discrepancies. However, it appears necessary to discuss the results which will be obtained with the optically thin approximation from that point of view (see last section).
4-5. The most intricate problem is to disentangle the role of solar EUV ionization and Ly $\alpha$ excitation anisotropies from those of the solar wind. The reason for treating together EUV and Ly $\alpha$ effects is that they should both induce correlated enhancements with solar activity and spatial distributions correlated with the solar active regions. In addition, in both cases the effect on one $\mathrm{H}$ atom is averaged over the full solar disk, which has a tendency to smooth out the solar irregularities. There are the following three distinct effects:

1. Independently of the hydrogen distribution, all the emissivities are proportional to the incident Ly $\alpha$ radiation intensity and thus departures from the "isotropic" model can be the "instantaneous" image of solar Ly $\alpha$ asphericities: longitudinal and latitudinal anisotropies are both partially smoothed out by a full disk averaging.

In fact, both longitudinal and latitudinal Ly $\alpha$ anisotropies do affect the intensity curve corresponding to a full scan plane. The different lines of sight starting from the point of observation contain atoms which are illuminated by different solar disks centered on different latitudes and longitudes. Let us assume that the intensity enhancement in north and south ecliptic directions for one observation is due to longitudinal anisotropies: i.e., the sun's surface does present a great amount of sunspots exactly in front of the earth and lower emissions at the other longitudes. All the atoms of the north and south lines of sight will benefit by this enhancement in emission, since they have the same longitude. Along the two opposite ecliptic lines of sight the far atoms are facing a less emissive disk.

There would be two consequences in this case. First, for some observations the earth would face low brightness solar

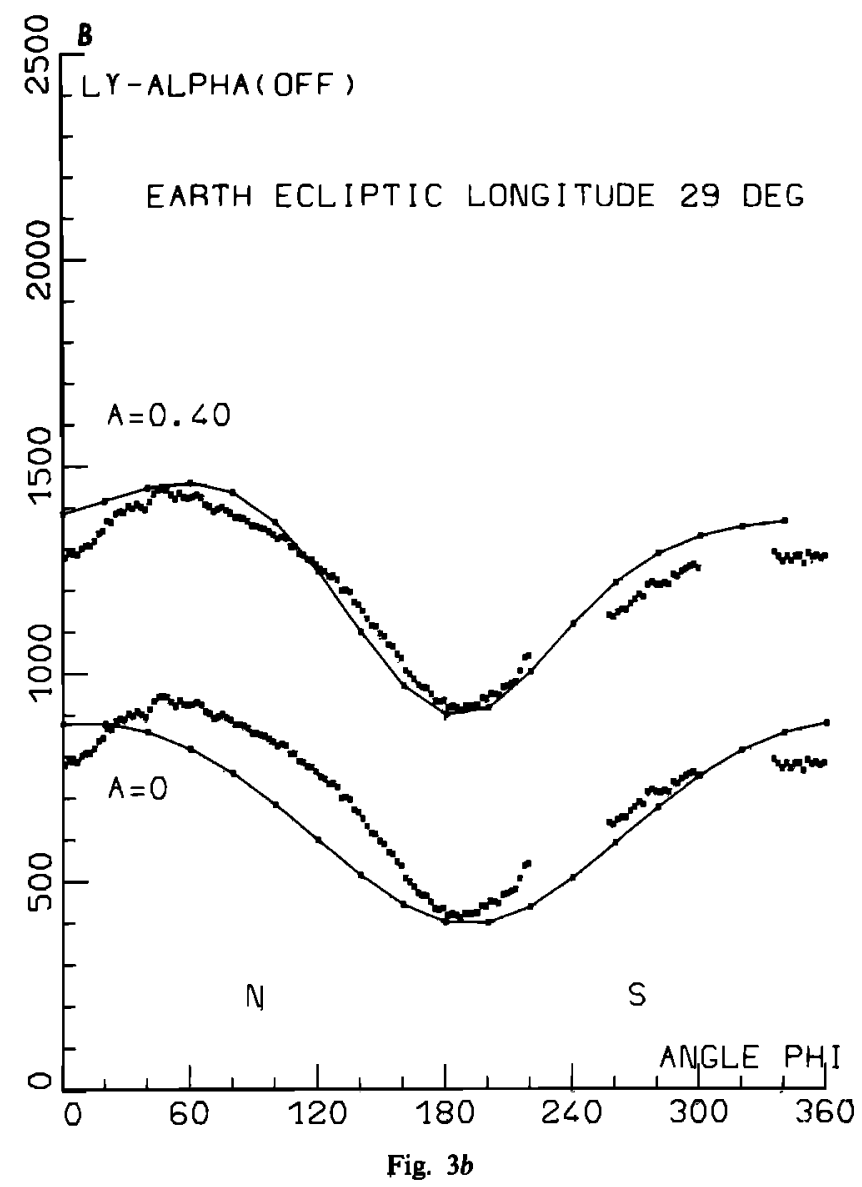


disk, inducing lower intensities for north and south directions. This is not at all observed. Second, the solar rotation would induce strong fluctuations of the mean intensity level from one observation to the other. Such fluctuations are not observed. The systematic effect measured of enhancement of the signal for north and south ecliptic direction could, however, better correspond to latitudinal variations of the solar $\mathrm{Ly} \alpha$ flux. However, that should mean a greater luminosity of the solar disk as viewed from an atom over the polar regions compared to an atom traveling near the ecliptic plane. Also, that is exactly contrary to what is expected from observations of solar active regions, which appear generally at low and midlatitudes. Thus we can no longer invoke a latitude effect of the resonance excitation Ly $\propto$ flux.

2. The Ly $\alpha$ radiation pressure acting on the atoms influences the bending of the trajectories. The present model assumes a permanent and stationary flow of gas, and thus a constant Ly $\alpha$ intensity characterized by the parameters $\mu$ (ratio of radiation pressure force to gravitational force). Spatial and temporal variations of the Ly $\alpha$ flux lead to departures from the model trajectories. However, owing to the respective time scales of the inflow and of the solar rotation (the mean velocity for an $\mathrm{H}$ atom is of $0.35 \mathrm{AU} / \mathrm{month}$ ), the dynamical effect of solar longitudinal anisotropies is timeaveraged. As for latitude variations, significant effects would have also introduced deviations from the model in the velocity distributions at high latitude, which should have been detected by the spectral study with the $\mathbf{H}$ absorption cell, but they were not [Bertaux et al., 1984]. In fact, if we were able to find a good agreement with the spectral modeling for one constant value of the parameter $\mu$, it suggests that the flow was not too

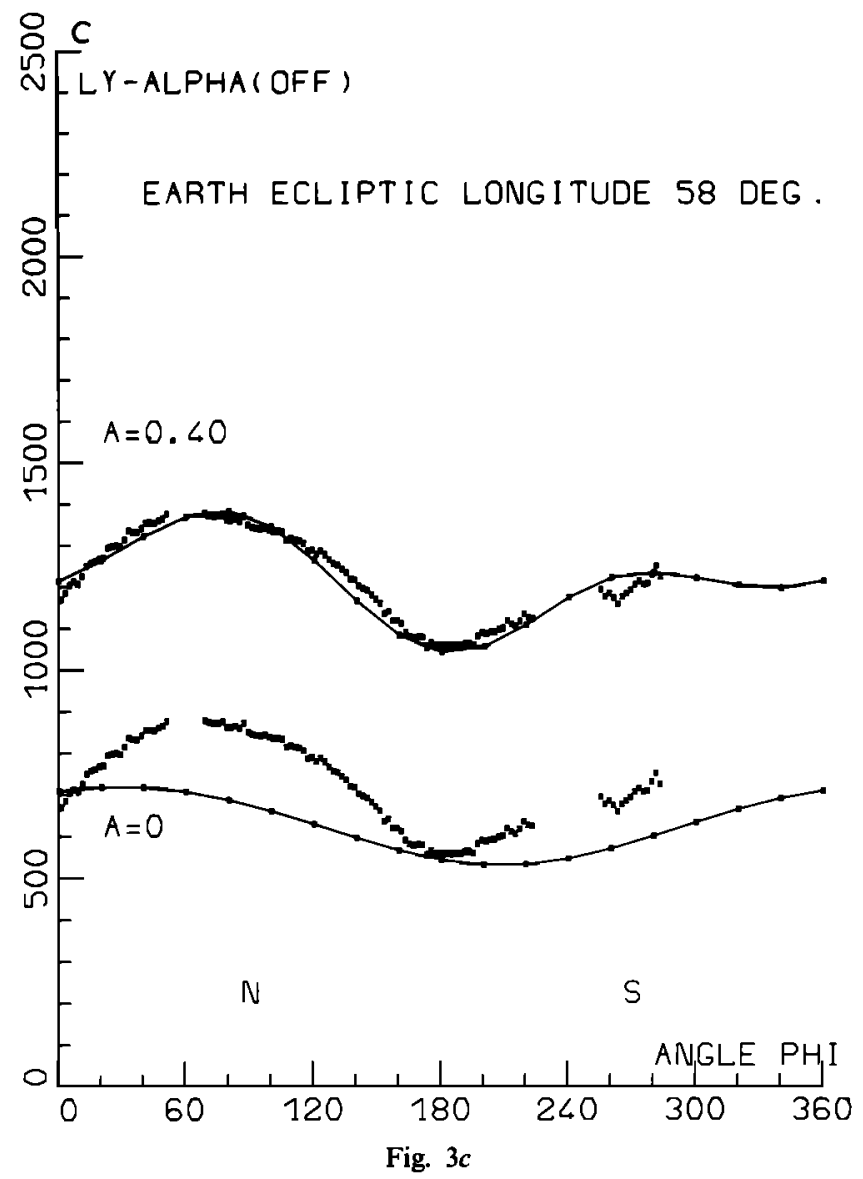

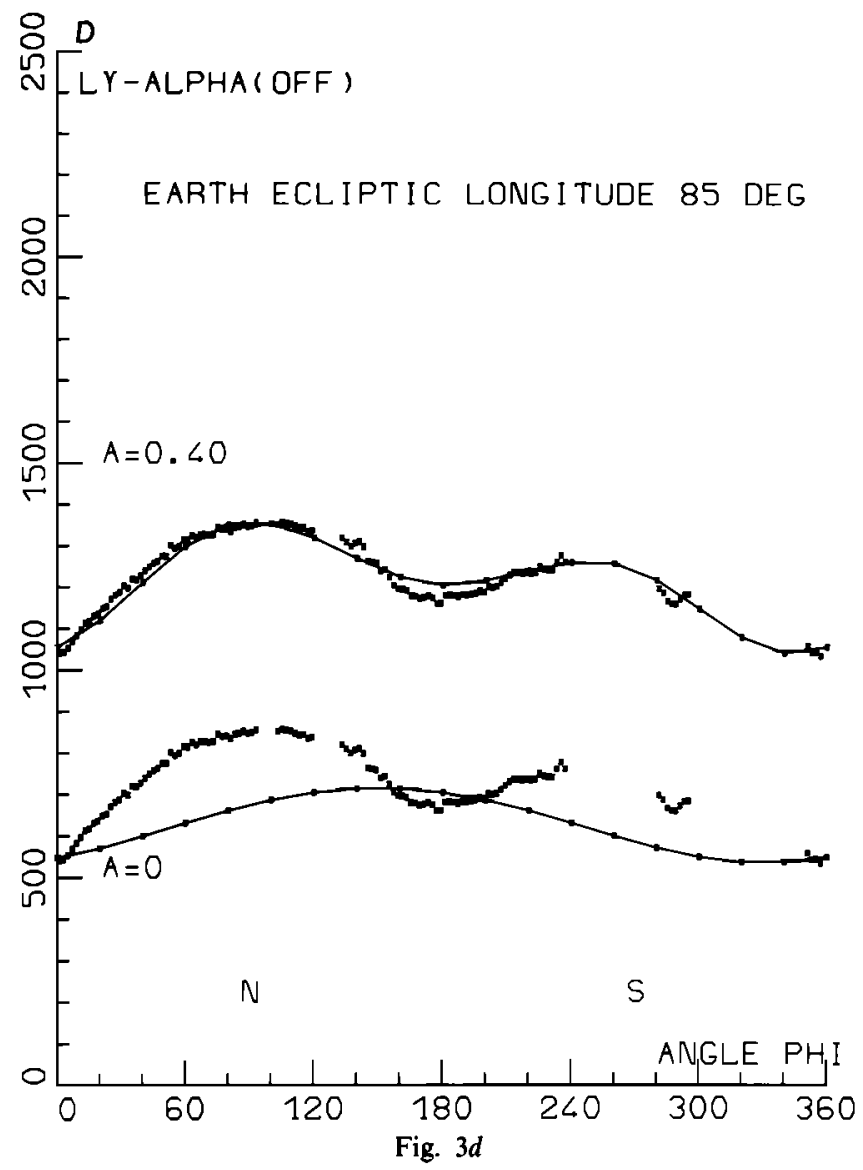

far from being stationary and that there was no strong variations of the solar Ly $\alpha$ flux during the one-year observation period.

3. The solar EUV flux, ionizing $H$ atoms at $\lambda \leq 91.2 \mathrm{~nm}$, is responsible for a minor contribution of the ionization processes. Only latitudinal variations have to be taken into account, since longitudinal effects are largely time averaged. If we assume a same pattern for variations of Ly $\alpha$ and EUV solar fluxes, then we can remark that the effects (1) and (3) are reversed, since a larger ionization rate in the near ecliptic regions reduces the density, whereas a greater incident Ly $\alpha$ flux in the same region enhances the emissivity.

Even by assuming very important differences for EUV photoionization rate with varying latitude, it appears quite impossible to explain the observations with such variations. Changes of the order of $40 \%$ for the densities are caused by changes of the same order of magnitude for the total ionizing rates. Since the EUV ionizations rate represents only $20 \%$ of the total ionization [Holzer, 1977], it would imply anisotropies of a factor of three at least of the photoionization rate, after full disk averaging, which is impossible.

At this point of the discussion it is apparent that the anisotropies in the solar Ly $\alpha$ flux cannot have the main role in the observed effects.

In addition, what is peculiar and fortunate in the interpretation of the results is that the Prognoz measurements have been recorded during a minimum solar activity period. In 1976-1977 the Zurich sunspot number $R z$ did not exceed 25. Thus there are only scarce and weak sunspots to produce strong latitude effects of the Ly $\alpha$ and EUV flux. A recent study of Cook et al. [1981], who have used statistical fre- 


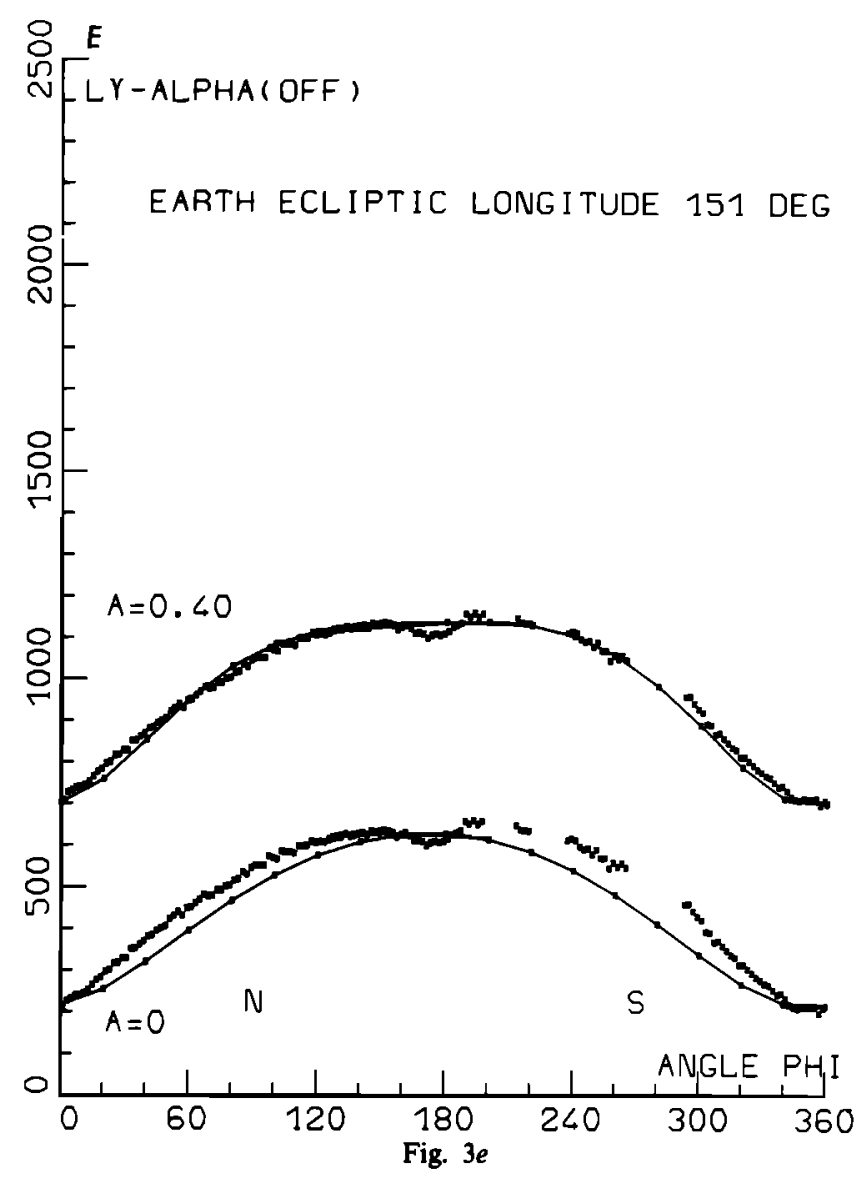

quencies of location and contrast of solar active regions, estimated at only a few percent the decrease of the disk averaged Ly $\alpha$ flux seen from an ecliptic point of observation to a polar one for low solar activity periods $\left(R_{z} \leq 80\right)$. Therefore we will neglect this type of solar anisotropies in the following model. This means that we will use the stationary model with a spherical Ly $\alpha$ incident flux. In addition, we will consider that the photoionization rate is independent of the latitude. This rate has been evaluated by Holzer [1977] to be about 0.9 $\times 10^{-7} \mathrm{~s}^{-1}$ at $1 \mathrm{AU}$ for periods of low activity.

6. The ionization by the solar wind is a directional effect: at a given point of the solar system the solar wind ionization is determined by the local solar wind properties, related through a tube of plasma trajectories, to a small portion of the solar surface, whereas photoionization is determined by a full solar disk.

Spatial relative variations of the solar wind fluxes are followed by relative variations of the solar wind ionization rate of the same order, which is also true for the global ionization rate, since the solar wind ionization is the most important loss process. From the three following ionizing effects of the solar wind

$$
\begin{gathered}
\mathrm{H}+\mathrm{H}^{+} \rightarrow \mathrm{H}^{+}+\mathrm{H} \\
\mathrm{H}+\mathrm{He}^{++} \rightarrow \mathrm{H}^{+}+\mathrm{He}^{+} \\
\mathrm{H}+\mathrm{e}^{-} \rightarrow \mathrm{H}^{+}+2 \mathrm{e}^{-}
\end{gathered}
$$

charge exchange between solar protons and atomic hydrogen is the main effect [Holzer, 1977].

The charge transfer with alpha particles has been evaluated to be about 200 times weaker than charge transfer with pro- tons as a result of both lower fluxes and lower cross sections [Feldman et al., 1977; Fite et al., 1960]. Ionization by impact with a solar electron has been calculated by Holzer [1977], who found $0.19 \times 10^{-7} \mathrm{~s}^{-1}$ at $1 \mathrm{AU}$, which is much less than the proton charge transfer rate of the order of $5 \times 10^{-7} \mathrm{~s}^{-1}$. Unlike the other processes, the electron collisional ionization rate cannot be considered as a $r^{-2}$ function of the radial heliocentric distance in the range of distances used in the model and decreases more rapidly. We are neglecting this fact in the modeling, assuming this is a minor effect; however, should also be noted that a variation of the mean velocity of the solar protons (such as the one already observed), independently of the plasma flux value, implies a variation of the charge exchange cross section (as will be discussed in the last section) and thus also a variation of the ionization rate.

\section{Prognoz Results and Anisotropic SOLAR WIND MODELING}

Only the Ly $\alpha$ intensity measurements obtained with the Lyman $\alpha$ photometer flown on Prognoz 5 and 6 in 1976 and

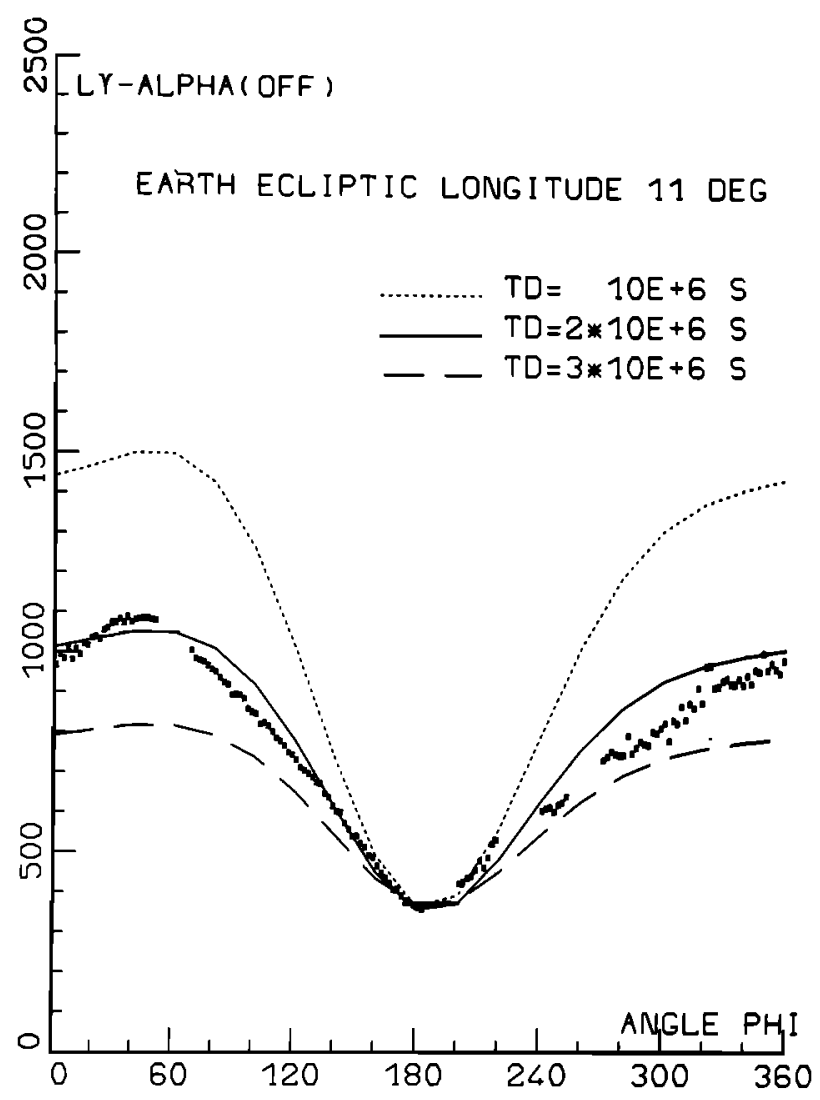

Fig. 4. Influence of the ionization rate value illustrated as an example for $\mathrm{S}_{1}$. While the relative decrease of the ionization rate from equator to solar poles has been fixed at a constant value $(A=0.40)$, here the varying parameter is the ionization rate in the solar equatorial plane $\beta_{E}$. The three corresponding lifetimes at $1 \mathrm{AU}$ in that plane, $T_{D}$, are indicated $\left(T_{D}=\beta_{E}{ }^{-1}\right)$. The three curves have been normalized to measurements for the downwind direction $\phi \simeq 180^{\circ}$, which means they would correspond to different values of the density at infinity. It is clear that the measured upwind to downwind intensity ratio precludes the extreme values used here for the ionization lifetime. However, it can be remarked that the direction of maximum intensity has been displaced from $\phi=0^{\circ}$ (isotropic model) to the same value $\left(\phi \simeq 60^{\circ}\right)$, whatever the value of $T_{D}$. Therefore the direction of maximum intensity is only related to the parameter $A$ and is a good indicator for this one. This is particularly true for seances $S_{1}$ to $S_{3}$, which are on the side of the wind flow. 
1977 are presented here. Spectral profile studies of the $H$ velocity field with the absorption cell were used to determine some parameters of the model $\left(V_{w}, T\right.$, and $\mu$ ). (J. L. Bertaux et al., unpublished observations, 1984). A description of the geometry has also been given in this paper. The five scan planes, $P_{1}$ to $P_{5}$, perpendicular to the sunearth line and the corresponding positions of the earth are shown in Figure 2 (letters $S_{1}$ to $\left.S_{5}\right)$. Two of them $\left(P_{3}\right.$ and $\left.P_{2}\right)$ are very near the downwind position where the strongest anisotropic effects are expected; two others $\left(P_{1}\right.$ and $\left.P_{5}\right)$ are in the lateral positions in respect to the sunwind axis, so that the scan planes contain upwind looking directions where the ionization effects are still very weak and the last position $\left(\mathbf{P}_{2}\right)$ is an intermediate one. In every scan plan the measured intensity is plotted in counts per second as a function of the spin angle $\phi$ (Figure 2) such defined that the direction $\phi=0^{\circ}$ is in the ecliptic plane, in the sense which is opposite to the earth's orbital motion; $\phi=90^{\circ}$ and $270^{\circ}$ are the north ecliptic and south ecliptic directions, respectively.

Deficiencies of data are due either to starlight contamination in the field of view or to a too important geocoronal contamination. The whole set of data were corrected from the exospheric hydrogen Ly $\alpha$ contribution according to a method explained by J. L. Bertaux et al. (unpublished manuscript, 1984). Here we do not use the directions of sight for which the relative contribution of the terrestrial Ly $\alpha$ as deduced from the method was larger than $10 \%$ of the total measured intensity geocoronal plus interplanetary). In this case the maximum relative error on the interplanetary Ly $\alpha$ emission intensity resulting from the geocoronal contamination is evaluated at 2-3\%.

Basically, the present anisotropic model is derived from the numerical model which was constructed for line profile calculations (R. Lallement and J. L. Bertaux, unpublished manuscript, 1984). The only difference is in the calculation of the extinction factors along each individual trajectory.

We first describe briefly the isotropic model. At infinity the velocity distribution of $\mathbf{H}$ atoms is described by a bulk velocity $V_{0}$ and a Maxwell-Boltzmann distribution at temperature $T$. Atoms are submitted to the apparent solar gravity, proportional to $1-\mu$. The values of the above parameters have been determined to be (J. L. Bertaux et al., unpublished manuscript, 1984):

$$
\begin{aligned}
T & =\left(8,000^{\circ} \pm 500^{\circ}\right) \mathrm{K} \\
V_{0} & =(20 \pm 1) \mathrm{km} \mathrm{s}^{-1} \\
\mu & =(0.75 \pm 0.10)
\end{aligned}
$$

and the direction of $\mathbf{V}_{0}$ has been found identical to the corresponding value for the flow of interstellar helium [Dalaudier et al., 1984]: ecliptic longitude $\left(74.5^{\circ} \pm 3^{\circ}\right)$ and ecliptic latitude $\left(-6^{\circ} \pm 3^{\circ}\right)$ in ecliptic coordinates.

The wind flow vector $V_{0}$ is opposite to $V_{w}$, which describes the motion of the sun in relation to the local interstellar medium.

In order to compute the density and the emissivity the relevant quantities obtained for the case of a monocinetic model (cold model) are integrated over the velocity space at infinity. A nonisotropic scattering function is introduced for $L y \alpha$ [Brandt and Chamberlain, 1959], and the solar Ly $\alpha$ spectral profile is assumed to be flat at its center, in the wavelength range of interest.

The present anisotropic model assumes an axial symmetry of the solar wind. The choice of the axis is now discussed. As it

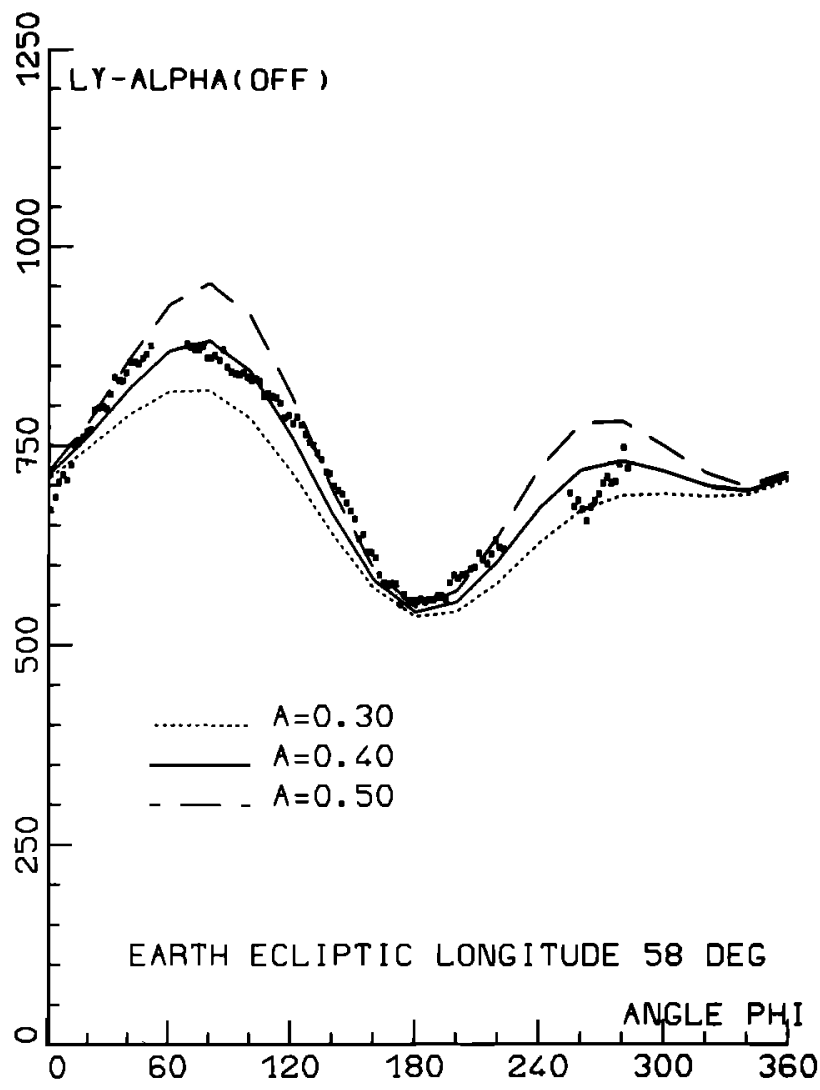

Fig. 5. Influence of the anisotropy $A$ coefficient, which represents the relative variation of the ionization rate when the heliographic latitude is changing from $0^{\circ}$ to $90^{\circ}$. The equatorial ionization rate has been fixed at $\beta_{E}=5 \times 10^{-7} \mathrm{~s}^{-1}\left(T_{D} \simeq 2 \times 10^{6} \mathrm{~s}\right)$. The contrast between the north or south ecliptic directions $\left(\phi=90^{\circ}\right.$ and $\left.\phi=270^{\circ}\right)$ and the ecliptic directions $\left(\phi=0^{\circ}\right.$ or $\left.180^{\circ}\right)$ is increasing with increasing $A$. The three models correspond to the same density at infinity, which means that for the ecliptic directions the intensity is not very sensitive to the value of $\beta$ at high heliographic latitudes once the equatorial value is fixed.

was mentioned earlier, there are enhancements of the emissivity in both the north and south ecliptic directions which suggest that the solar wind axis is not far from being normal to the ecliptic plane. As it is known, the sun spin axis is only slightly tilted from the normal to ecliptic. From a physical point of view it is likely that the solar axis of rotation will have a special role. However, the properties of the solar wind are supposed to be strongly related to the solar magnetic features and could as a consequence present a symmetry around an instantaneous magnetic axis. Such an axis could be significantly tilted from the solar rotation axis [Hundhausen, 1978]. However, since the loss processes of the hydrogen are time-averaged over several solar rotations, what is observed is the effect of the mean spatial properties of the solar wind, also averaged over at least a whole solar rotation. In the case of a tilted symmetry axis, it describes a cone which axis is the rotation axis, and the final result is a symmetry of the ionization effects around this rotation axis. The exact relative positions of the equatorial plane (or of the rotation axis) and the ecliptic plane as well as the $6^{\circ}$ inclination of the wind velocity vector $\mathbf{V}_{w}$ on the ecliptic plane were taken into account in the present model. The corresponding geometry is shown on Figure 7.

The whole ionization rate including photoionization and solar wind effects is assumed to have a $r^{-2}$ radial dependence 


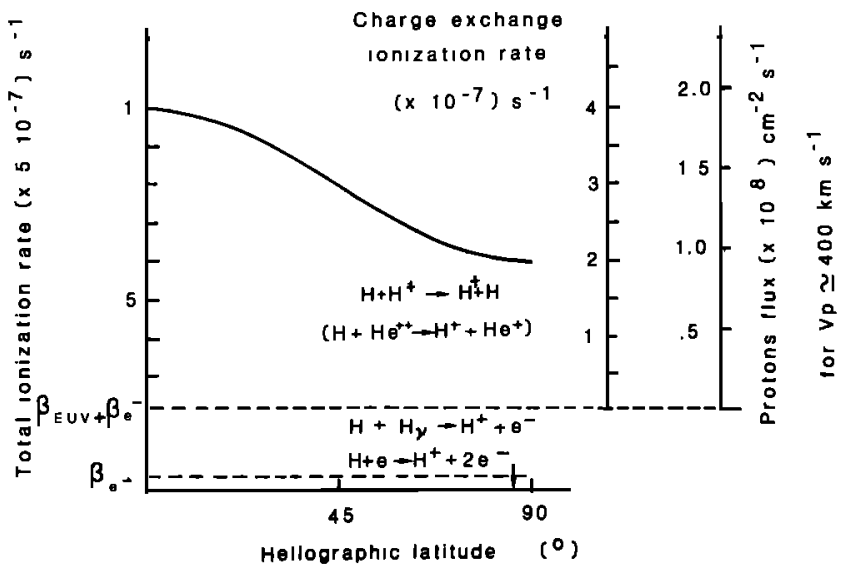

Fig. 6. Heliographic dependence of the global ionization rate (at $1 \mathrm{AU})$ which corresponds to the adjustment presented in Figures 3-5. This is the simple trigonometric function $\beta=5.0\left[1-0.4 \sin ^{2} \lambda\right]$ $10^{-7} \mathrm{~s}^{-1}$. If one neglects the latitudinal variations of the electron collisional ionization rate (which is here a very minor effect) and assumes also a constant photoionization [Holzer, 1977], which is quite reasonable for a low solar activity [Cook et al., 1981], it provides a relative decrease of about $50 \%$ for the charge exchange rate. Since the charge transfer with alpha particles is about 200 times weaker than for the protons, the rate variation can be interpreted in terms of a proton flux variation, provided that a heliographic variation of the mean solar wind velocity is assumed. Results are presented for a constant solar wind velocity $V=400 \mathrm{~km} \mathrm{~s}^{-1}$ (right scale).

and to be related to the heliographic latitude $\lambda$ so that

$$
\beta(r, \lambda)=\beta_{0} r^{-2}\left(1-A \sin ^{2} \lambda\right)
$$

where $\beta_{0}$ represents the ionization rate at $1 \mathrm{AU}$ from the sun in the solar equatorial plane, $r$ is the radial distance in AU units, and $A$ is a parameter $(0 \leq A \leq 1)$ describing the degree of anisotropy. The $\beta_{0}$ and the $A$ are the two parameters which we propose to derive from a comparison with the data.

Calculations of the extinction factor at a fixed point $M$ for every trajectory followed by an atom from "infinity" to $M$ are presented in the appendix.

The use of a trigonometric function for the latitude dependence was first employed by Kumar and Broadfoot [1978] and presents the advantage that it can be analytically integrated along one trajectory (see the appendix).

For our Prognoz measurements the determination of $\beta_{0}$ and $A$ was made by a visual adjustment of the model to the data simultaneously for the five observations. Generally speaking, changes in $\beta_{0}$ correspond to a global increase or decrease of the ionization rate in all space and thus to a variation of the negative gradient of $\mathrm{H}$ density along the flow from upwind to downwind, whereas the parameter $A$ measures the asphericity. One could think of two distinct adjustments. In fact, owing to the great velocity dispersion, filling in of the downwind region comes from atoms which have traveled at all heliographic latitudes, whatever the latitude of the observed direction.

A good agreement could be finally found between the model and the data simultaneously for the five observations, as can be seen in Figures $3 a-3 e$. The final result is $\beta_{0}=(5.0 \pm 0.50)$ $10^{-7} \mathrm{~s}^{-1}$ and $A=0.40 \pm 0.10$, which means a $30-50 \%$ decrease in the ionization rate over the solar pole in comparison with the equatorial plane.

Figures $3 a-3 e$ correspond to observations $S_{1}$ to $S_{5}$, respectively, where the distribution of Ly $\alpha$ intensity was recorded in planes perpendicular to the earth-sun line at positions de- scribed by their ecliptic longitude: $11^{\circ}, 29^{\circ}, 58^{\circ}, 85^{\circ}$, and $151^{\circ}$ for observations $S_{1}$ to $S_{5}$, respectively.

In each figure the intensity measurements (data points) are represented as a function of spin angle $\phi$. They are plotted twice, with a vertical shift. On the bottom curve they are compared to a model assuming isotropy of the solar wind, whereas on the top curve they are compared with our anisotropic model characterized by $A=0.40$.

The letters $\mathbf{N}$ and $\mathbf{S}$ indicate directions toward north and south ecliptic, respectively. The data differ from the isotropic model in three respects: (1) there is more intensity in the data toward the north and south ecliptic region; (2) the maximum intensity of the model is found at $\phi=0^{\circ}$ for observations $S_{1}$ to $S_{3}$, (Figures $3 a-3 c$ ), whereas the data maximum is found around $\phi=60^{\circ}$; (3) for observations $S_{3}$ and $S_{4}$ near the downwind direction (Figures $3 c$ and $3 d$ ) there are two maxima instead of one in the model.

Even in Figure $3 e$, where there are only subtle differences between the two models, the anisotropic model fits still better with the data.

The departure between the two models is more pronounced for observations near the downwind axis (Figures $3 c$ and $3 d$ ), as is expected from the cumulative effect of the anisotropy as explained on Figure 1.

In Figure 4 the data of observation $S_{1}$ (which probes both the upwind and the downwind regions) are compared to three anisotropic models corresponding all to the same parameter $A$ but with different values of $\beta_{0}$. The contrast for intensity between the downwind and upwind directions is strongly dependent of $\beta_{0}$. On the figure the lifetime $T_{D}=\beta_{0}{ }^{-1}$ is indicated.

However, the position of the maximum is insensitive to $\beta_{0}$ and is only related to the value of $A$.

In Figure 5 the data of observations $S_{3}$ (downwind plane nearly perpendicular to $V_{0}$ ) are compared to three anisotropic models with the same value of $\beta_{0}$ and different values of the coefficient $A$.

A visual estimate for Figures 4 and 5 together with a similar exercise on the three other observations (not shown here) allows the determination of the possible range of values for the parameters $\beta_{0}$ and $A$ quoted above.

\section{Discussion}

Without any doubt, our measurements provide a clear confirmation of the phenomenon which was already observed by Kumar and Broadfoot [1978] from 1974 Mariner 10 data. The solar wind anisotropies are far from being a minor effect, at least at the time of the two observations. The reasons for which we were able to present a better adjustment of model to data are now briefly discussed.

From an experimental point of view the Prognoz results are characterized by a much better statistical accuracy and by a more detailed angular scanning (each point plotted on Figures $3 a-3 e$ for a bin of $\Delta \phi=2^{\circ}$ corresponds to an average of at least 25 measurements, with the average counting rate being $\simeq 600$ counts/measurement yielding a $1 \%$ statistical accuracy).

Concerning the geometry of the observations, it should be noted that the three Mariner 10 angular scans were perfectly chosen to provide the most important departures from the isotropic model, since they were performed when Mariner 10 was in the downwind region. In fact, they correspond to our $P_{3}$ and $P_{4}$ seances. As we have already explained, for a more convincing study we decided to use also additional observations made from as most as possible upwind positions, where 
differences between data and isotropic model should be smaller, and they are indeed.

Concerning the modeling, we have constructed a much more realistic model than those used by the two groups which analyzed independently the same Mariner 10 data [Kumar and Broadfoot, 1978, 1979; Witt et al., 1979]. Instead of a quasi-monocinetic model (they assumed that all atoms were flowing at the same velocity) we took completely into account the thermal velocity dispersion at infinity, and this changes substantially the density results. We also used a more appropriate geometry (by using an axial symmetry around the true solar rotation axis instead of the ecliptic axis and introducing the small declination of the interstellar wind). In addition, we used a realistic set of dynamical parameters $\left(T, \mathrm{~V}_{w}\right.$, and $\left.\mu\right)$ since they were determined from our spectral study (J. L. Bertaux et al., unpublished manuscript, 1984), whereas they were only estimated in the Mariner 10 interpretation.

From the Mariner 10 data the degree of anisotropy $A$ was found to be $A \simeq 0.5$ by Kumar and Broadfoot [1978, 1979] and $A \simeq 0.30$ by Witt et al. $[1979,1981]$, from the same set of data. It appears that the degree of anisotropy $A$ is quite comparable for the Mariner 10 and for the Prognoz periods of observations (with the restriction however that the determination with a cold model is approximate in the case of Mariner 10). Since the hydrogen distribution observed by Mariner 10 in 1974 is the result of the anterior ionization back to 1973 , and since the last observations by Prognoz were made in December 1977 , we conclude with the persistence of the large degree of anisotropy during the whole period 1973-1977, which corresponds in fact to the 5 years of minimal solar activity for the last solar cycle. Thus the anisotropic pattern is far from being an occasional phenomenon.

What is really measured is the decrease of the total ionization rate, essentially EUV photoionization and charge exchange with the solar wind protons. We will now briefly discuss the implications of such a latitudinal decrease in terms of solar wind mass flux.

As it was shown by the Cook et al. [1981] calculations, for a low solar activity period the solar line center Ly $\alpha$ flux at a given point, averaged over the full disk, is constant within a few percent when changing the latitude of the point. As we have said, all the atomic hydrogen which contributes to the Prognoz signal has been submitted to the ionizing influence of a low activity sun [1975-1977]. For this period the sunspot number was less than 25 , and an evaluation using the Cook et al. results gives a relative variation for the fluxes within and above ecliptic plane of about $3 \%$ at line center and $\simeq 2 \%$ for the integrated line. If we consider that the solar EUV is strongly related to the $\mathrm{Ly} \alpha$ (they are both correlated with the

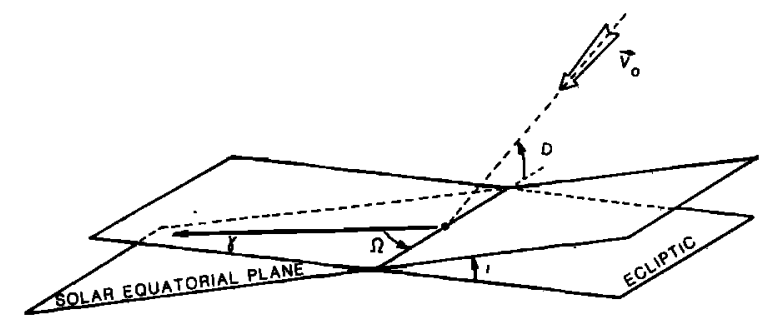

Fig. 7. Relative positions of the interstellar wind velocity vector and the solar equatorial plane. By coincidence, the direction of the relative velocity vector of the hydrogen flow with respect to the solar system has almost exactly the same ecliptic longitude as the ascending node of the solar equatorial plane. The used values are $\Omega=75^{\circ}$, $i=7.2^{\circ}$, and $D=6^{\circ}$.

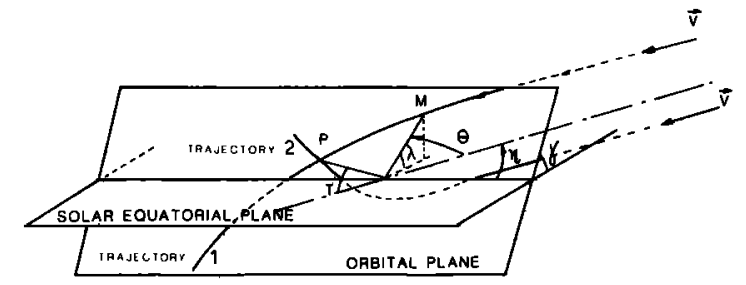

Fig. 8. Example of the two trajectories which lead to a given point $P$ for a given velocity vector $V=V_{0}+V_{T}$ if $V_{0}$ is the bulk velocity and $V_{T}$ the thermic velocity. The loss factor at point $P$ depends on the evolution of both the distance to sun $r$ and the heliographic latitude $\lambda$ along the trajectory (as indicated for a current point $M$ ). The latitude $\lambda$ can be calculated in terms of the three angles $T, \eta$, and $\gamma$.

indexes of solar activity) then the EUV ionization rate is almost constant with varying latitude (also within 3\%). Thus the latitudinal changes of the ionization are almost entirely due to the charge exchange with solar wind protons. For low solar activity the EUV photoionization has been estimated to represent about $12 \%$ of the total loss process [Holzer, 1977] but for an estimated global rate of about $7.510^{-7} \mathrm{~s}^{-1}$ at 1 AU. In fact, we find here a smaller global rate of about $510^{-7}$ $s$ in the equatorial plane. If we assume the same photoionization rate, it thus represents $22 \%$ of the whole rate. However, there is the small contribution to losses of the collisions with solar electrons. It is not important to neglect possible anisotropies of this effect. Thus together with the photoionization effect we will consider that there is an isotropic part of the ionization effect with a $10-25 \%$ contribution to the whole rate.

As a consequence, assuming as was discussed above the independence of the photoionization rate with latitude, we find that a $40 \%$ decrease of the global rate corresponds to $44-53 \%$ of latitudinal decrease of the charge exchange rate.

The charge exchange rate is proportional to the quantity $\sigma\left(V_{p}\right) F_{P}$, where $\sigma$ is the charge exchange cross section, and $F_{p}=n_{p} V_{p}$ is the proton flux at one $\mathrm{AU}\left(n_{p}\right.$ is the density and $V_{p}$ is the velocity of the protons relative to the neutral hydrogen). Since the solar proton velocity $\left(400 \mathrm{~km} \mathrm{~s}^{-1}\right)$ is much larger than the hydrogen velocity $\left(\simeq 20 \mathrm{~km} \mathrm{~s}^{-1}\right)$, we can consider that the relative velocity is the solar wind velocity. These expressions are related to the fact that we have assumed a radial decrease as $r^{-2}$ of the ionizing rates, implied by a $r^{-2}$ decrease of the density and a constant velocity of the protons (heliocentric distances of more than 0.5 AU are only of importance here).

The velocity dependence of the charge exchange cross section has been measured by Fite et al. [1960] for the range of proton energy corresponding to the solar wind. Indeed the velocity dependence is such that the observed velocity variations of the solar protons with latitude are of importance. As a consequence, the conclusions about the latitudinal decrease of the proton flux corresponding to a given latitudinal decrease of charge exchange ionization strongly depend on the assumed velocity field. From the work by Fite et al. [1960] it can be calculated that changes of the mean velocity from $\mathbf{4 0 0}$ $\mathrm{km} \mathrm{s}^{-1}$ at low latitudes to $800 \mathrm{~km} \mathrm{~s}^{-1}$ at high latitudes correspond to a decrease of $25 \%$ of the charge exchange cross section.

In this extreme case the observed decrease described by $A=0.40$ corresponds to a decrease of the mean corpuscular proton flux of $25-35 \%$ from latitude zero to above the poles.

On the contrary, for a spherical velocity field the decrease of 
the ionization rate is directly proportional to the flux decrease (about 50\%).

The adjusted heliographic dependence of the total ionization rate, the evaluated charge exchange rate, and the corresponding protons fluxes in the particular case of a spherical velocity field are shown in Figure 6.

An important point is that the Ly $\propto$ mapping allows us to determine, even with an uncalibrated instrument, the absolute value of the mean ionization rate, and the absolute value of the solar wind flux can be derived. From the measured ratio of intensities in the upwind and downwind directions the equatorial mean total ionization rate was found to be $(5 \pm 0.5)$ $\times 10^{-7} \mathrm{~s}^{-1}$ when our single scattering model is used. Taking into account a $10-25 \%$ contribution of the photo and electron collisional ionization rates we derive a charge exchange rate of $(3.3-5) \times 10^{-7} \mathrm{~s}^{-1}$, corresponding for a mean velocity of $400 \mathrm{~km} \mathrm{~s}^{-1}$ to a mean equatorial proton flux of $(1.6-2.4)$ $\times 10^{8} \mathrm{~cm}^{-2} \mathrm{~s}^{-1}$, which is substantially lower than the currently admitted average values of $3.3 \times 10^{8}$ protons $\mathrm{cm}^{-2} \mathrm{~s}^{-1}$ [Feldman et al., 1977; Hundhausen et al., 1970].

However, multiple scattering will affect the upwinddownwind intensity ratio. Keller et al. [1981] computed that for a density at infinity of $0.05 \mathrm{~cm}^{-3}$ very near the Prognoz value), multiple scattering effects decrease this ratio by about $30 \%$ in respect to the single scattering approximation.

By using this result and our single scattering model we can evaluate that the charge exchange rate could be at maximum underestimated by about $50 \%$. This leads to an equatorial proton flux of $(2.4-3.6) \times 10^{8} \mathrm{~cm}^{-2} \mathrm{~s}^{-1}$, now consistent with the "classical" estimate [Schwenn, 1982]. However, from the present point of view of the solar wind anisotropies it is important to notice that these corrections do not change much the value of the coefficient $A$. In fact, since the multiple scattering effects tend to "smooth out" the differences between the intensities in different directions corresponding to different densities, taking into account the multiple scattering effects would lead to a value of $A$ only slightly greater than the present one.

In conclusion, it has been shown that the interplanetary Ly $\alpha$ mapping provides a good diagnostic for the out-of-ecliptic large-scale properties of the solar wind. This becomes especially true now, since the interstellar wind parameters are better known and the modeling is more realistic. Of course, it would be of great interest to have such a monitoring of the solar wind anisotropies for a full solar cycle and eventually to search for a correlation of these low polar fluxes with the presence of polar coronal holes. Detailed studies of such polar low density holes, as was made by Munro and Jackson [1977], are of great interest and could be considered again with the present new value of the solar wind flux at high latitudes as an upper boundary condition to their model of solar wind expansion in the corona.

Finally, it should be noted that the low-ecliptic inclination of the interstellar wind, which is totally fortuitous, is quite favorable to such a study. If the wind were coming from a high-latitude region the solar wind anisotropy would be much less clearly "printed" on the distribution of atomic hydrogen in the solar system.

\section{APPENDIX}

The following approached values for defining the position of the solar equatorial plane have been used: $\Omega=75.0^{\circ}$ (ecliptic longitude of the ascending node) and $i=7.2^{\circ}$ (inclination on the ecliptic). The ecliptic component of the wind velocity
$V_{w}\left(\lambda_{w}=254^{\circ}\right)$ has almost exactly the same direction than the ascending node (Figure 7).

For a given point $P$ and a given initial velocity vector "at infinity," $V$, there are two trajectories for the $\mathbf{H}$ atoms. Along each of these the decrease of the density inside the corresponding tube of trajectories is integrated to yield the "loss factor" at point $P$.

During the time interval $d t$, if the density is $n$ and the local ionization rate $\beta$, then the density variation is $d n=-n \beta(t) d t$ and the loss factor (the ratio of the density to the initial one) is $L=\exp \left(-\int_{\infty}^{p} \beta(t) d t\right)$.

By using as new variable, instead of the time $t$, the angular parameter $\theta$, whose origin is the asymptotic direction through the relation of conservation of angular momentum, it follows:

$$
L=\exp \left(-\frac{\beta_{0} r^{2} \theta_{i}}{p_{l} V}\right)
$$

where $p_{t}$ is the impact parameter, $V$ the velocity at infinity, $r_{0}=1 \mathrm{AU}, \beta_{0}$ the ionization rate at $1 \mathrm{AU}$, and $\theta_{i}(i=1,2)$ the angular parameter at point $M$ for the two types of trajectory.

If $T$ is the angle between $\mathbf{V}$ and the radial direction $\mathbf{S} P$, then $\theta_{1}=\pi-T$ (direct trajectory) and $\theta_{2}=\pi+T$ (indirect trajectory).

For the anisotropic model the ionization rate at a current point $M$ (Figure 8) along the trajectory is a function of the radial distance $r$ and of the heliographic latitude $\lambda$ of $M$ $\beta(M)=\beta_{0} r^{-2}\left(1-A \sin ^{2} \lambda\right)$. Thus

$$
L=\exp -\left(\frac{r_{0}{ }^{2} \beta_{0} \theta_{i}}{p_{i} V}\right) \exp \frac{r_{0} \beta_{0} A}{p_{i} V} \int_{0}^{\theta_{i}} \sin ^{2} \lambda d \theta
$$

The first term is the loss factor for the isotropic model $L_{0}$. If $\gamma$ is the angle between the orbital plane of the $\mathrm{H}$ atom and the equatorial solar plane (which intersect along the vector $\mathbf{u}$ ), then geometrical considerations give $\sin \lambda=\sin \gamma \sin (\eta+\theta)$, where $M$ is the angle between $V$ and $U$.

After integration along the trajectories we obtain

$$
\begin{gathered}
L_{(1,2)}=L o \exp \left(\frac{\beta_{0} \theta_{i}}{p_{i} V} \frac{A \sin ^{2} \gamma}{2} r_{0}{ }^{2}\right. \\
\left.\cdot\left(\begin{array}{c}
1 \\
1 \\
+(2)
\end{array} \frac{\sin 2(\eta-T)-\sin 2 \eta}{2 \theta_{i}}\right)\right)
\end{gathered}
$$

The sign is changing according to the type (1) or (2) of trajectory.

In order to compute the density at any given point in the solar system the full velocity distribution due to thermal dispersion was integrated (classical "hot" model, modified here for latitudinal variation of the ionization rate).

Acknowledgments. This experiment was a cooperative effort between the Laboratory of Ultraviolet Astronomy at the Space Research Institute (IKI) of the Academy of Science of USSR in Moscow and Service d'Aéronomie du CNRS in France. We are particularly grateful to H. Mironova, F. Semelin, and J. F. Brun for their long dedication to this experiment, and to J. C. Vial for useful discussions. This work was supported by Centre National d'Etudes Spaciales (France) under contracts CNES 83-201 and 84-201.

The Editor thanks P. W. Blum and another referee for their assistance in evaluating this paper.

\section{REFERENCES}

Bertaux, J. L., and R. Lallement, Analysis of interplanetary Lymanalpha line profile with a hydrogen absorption cell: Theory of the Doppler angular spectral scanning method, Astron. Astrophys., 140, $230,1984$. 
Bertaux, J. L., J. E. Blamont, N. Tabarie, V. G. Kurt, M. C. Bourgin, A. S. Smirnov, and N. N. Dementeva, Interstellar medium in the vicinity of the sun: A temperature measurement obtained with Mars-7 interplanetary probe, Astron. Astrophys., 46, 19, 1976.

Brandt, J. C., and J. W. Chamberlain, Interplanetary gas, I, Hydrogen radiation in the night sky, Astrophys. $J ., 130,670,1959$.

Coles, W. A., and S. Maagoe, Solar wind velocity from IPS observations, J. Geophys. Res., 77, 5622, 1972.

Cook, J. W., R. R. Meier, G. D. Bruekner, and M. E. Van Hoosier, Latitudinal anisotropy of the solar far ultraviolet flux: Effect on the Lyman-alpha sky background, Astron. Astrophys., 97, 394, 1981.

Dalaudier, F., J. L. Bertaux, V. G. Kurt, and E. N. Mironova, Characteristics of interstellar helium observed with Prognoz $658.4 \mathrm{~nm}$ photometer, Astron. Astrophys., 134, 171, 1984.

Feldman, W. C., S. J. Asbridge, S. J. Bame, and J. T. Gosling, Plasma and Magnetic Fields From the Sun, Solar Output and Its Variations, p. 351, edited by $\mathbf{O}$. R. White et al., Colorado University Associated Press, Boulder, 1977.

Fite, W. L., R. F. Stebbings, D. G. Hummer, and R. T. Brackman, Ionization and charge transfer in proton-hydrogen atom collisions, Phys. Rev., 119, 663, 1960.

Holzer, T. E., Neutral hydrogen in interplanetary space, Rev. Geophys. Space Phys., 15, 467, 1977.

Hundhausen, A. J., Solar wind spatial structure: The meaning of latitude gradients in observations averaged over solar longitude, $J$. Geophys. Res., 80, 4186, 1978.

Hundhausen, A. J., S. J. Bame, J. R. Asbridge, and S. J. Sydorisak, Solar wind protons properties Vela 3 observations from July 1965 to June 1967, J. Geophys. Res., 75, 4643, 1970.

Hundhausen, A. J., R. T. Hansen, and S. F. Hansen, Coronal evolution during the Sunspot cycle: Coronal holes observed with the $\mathbf{M}$ auna Loa K-coronameters, J. Geophys. Res., 86, 2079, 1981.

Joselyn, J. A., and T. E. Holzer, The effect of assymetric solar wind on the Lyman alpha sky background, J. Geophys. Res., 80, 903, 1975.

Keller, H. U., K. Richter, and G. E. Thomas, Multiple scattering of solar resonance radiation in the nearby interstellar medium, Astron. Astrophys., 102, 415, 1981.

Kumar, S., and A. L. Broadfoot, Signatures of solar wind latitudinal structure in interplanetary Lyman-alpha emissions: Mariner $10 \mathrm{ob}-$ servations, Astron. Astrophys., 69, L5, 1978.

Kumar, S., and A. L. Broadfoot, Evidence from Mariner 10 of solar wind flux depletion at high ecliptic latitudes, Astrophys. J., 228, 302, 1979.

Lallement, R., L'hydrogène atomique d'origine interstellaire dans le système solaire, Thèse de 3ème cycle, Université Paris VI, 1983.

Lallement, R., J. L. Bertaux, and F. Dalaudier, Interplanetary Lymanalpha spectral profiles and intensities for both repulsive and attractive solar force fields: Predicted absorption pattern by a hydrogen cell, Astron. Astrophys., in press, 1985.

Munro, R. H., and B. V. Jackson, Physical properties of a polar coronal role from 2 to $5 R_{0}$, Astrophys. J., 213, 874, 1977.

Rhodes, E. J., and E. J. Smith, Further evidence of a latitude gradient in the solar wind velocity, J. Geophys. Res., 81, 5833, 1976.

Schwenn, R., The "average" solar wind in the inner heliosphere: Structures and slow variations, Solar wind Five, NASA Conf. Publ., $2280,489,1982$.

Thomas, G. E., and J. E. Blamont, Galactic lyman alpha emission in the solar vicinity, Astron. Astrophys., 51, 283, 1976.

Witt, N., J. M. Ajello, and P. W. Blum, Solar wind latitudinal variations deduced from Mariner 10 interplanetary H (1216 $\AA$ ) observations, Astron. Astrophys., 73, 272, 1979.

Witt, N., J. M. Ajello, and P. W. Blum, Polar solar wind and interstellar wind properties from interplanetary Lyman-alpha radiation measurements, Astron. Astrophys., 95, 80, 1981.

Zhao, $X$., and A. J. Hundhausen, Spatial structure of solar wind in 1976, J. Geophys. Res., 88, 451, 1983.

J. L. Bertaux and R. Lallement, Service d'Aéronomie, du CNRS, Boite Postale 3, 91370 Verrieres le Buisson, France.

V. G. Kurt, Space Research Institute, Academy of Science of USSR, 84/32 Profsoyouznaia, Moscow, 117810, USSR.

(Received June 6, 1984;

revised August 23, 1984;

accepted September 12, 1984.) 